\title{
Simultaneous Development of SCC of the head and BCC of the leg: Successful Surgical Approach in Bulgarian patient
}

\author{
Dr Nikhil Oliveira ${ }^{1}$ and Prof Dr Georgi Tchernev ${ }^{2^{*}}$ \\ ${ }^{1}$ Student, Faculty of Medicine, Medical University of Sofia, Bulgaria \\ ${ }^{2}$ Onkoderma - Clinic for Dermatology, Venereology and Dermatologic Surgery, Sofia, Bulgaria
}

\begin{abstract}
Received: March 30, 2021; Accepted: April 02 2021; Published: April 12, 2021
*Corresponding author: Pro Dr. Georgi Tchernev, Onkoderma-Clinic for Dermatology, Venereology and Dermatologic Surgery

General Skobelev 26, 1606 Sofia.GSM:00359885588424; E-mail: georgi_tchernev@yahoo.de
\end{abstract}

Non-melanoma skin cancer (NMSC) is one of the most common cutaneous malignancies, with an ever-increasing incidence rate worldwide [1]. Studies have shown an association between intermittent and chronic sunlight exposure and the development of BCC and SCC respectively [2]. UV radiation is a known carcinogen that can induce mutations in the p53 gene, known also as the guardian of the genome [3]. This can lead to persistent damage and failure to eliminate dysplastic cells as subsequent mechanisms like p21 and p16 activation fail to arrest cell cycle. Proapoptotic proteins such as BAK and BAX are also unable to activate programmed cell death, ultimately leading to the formation of precancerous lesions and NMSC [3, 4].

An 85-year-old Caucasian male presented to the clinic with multiple actinic keratoses alongside a suspicious tumour-like lesion on his head as well as a further lesion on his right lower leg. A thorough patient history revealed chronic sun exposure without adequate skin protection throughout his lifetime. The lesions were thought to appear 7-10 years prior and persisted to date. Patient reported no immunodeficiencies or genetic abnormalities. No other concomitant disease, medication or significant information was noted in the patient history. Upon examination the actinic keratoses were all over the mid-scalp, crown (Fig. 1a \& 1b), preauricular (Fig. 1c) and periauricular (Fig. 1d) regions and were treated successfully with cryotherapy (Fig. 2f). The subsequent two lesions (Fig. 1f \& 3a) were suspected for tumours and were treated surgically via elliptical excisions (Fig. 2a \& 3a) without preliminary biopsies. The surgical excisions were made under local anaesthesia and performed with clear margins (Fig. 2b-2d \& 3a-3c) Histopathology confirmed squamous cell carcinoma (SCC) in the scalp as well as basal cell carcinoma (BCC) in the right lower leg respectively. A further work up was conducted to assess cervical, occipital, axially and inguinal lymph nodes however no metastatic spread was detected. A postoperative 21-day follow up (Fig. 2f) revealed successful closure of wound edges and no further signs of neoplasm.

In the presenting case, it is evident the patient's advanced age and chronic exposure of UV radiation has played a role in the development of premalignant actinic keratoses alongside the SCC lesion on his scalp.

There are number of known risk factors for developing multiple cutaneous neoplasms including hereditary disorders such as albinism or xeroderma pigmentosum [5-7]. Individuals with Xeroderma Pigmentosum (XP) are particularly susceptible to aggressive SCC of the scalp, in addition to other multiple neoplasms, due to the lack of DNA repair and photosensitivity $[6,7]$. However, with XP and other hereditary conditions, the neoplastic lesions (with excessive sun exposure) present at a much younger age due to lack of innate protective mechanisms and accelerated development of the neoplasms $[6,7]$. Furthermore, the clinical presentation is incredibly severe in these cases due to the aggressive malignant transformation showing the significant risk hereditary conditions possess in the predisposition of skin cancers [5-7]. Our patient showed no signs of aggressive malignant growth with just two isolated synchronous lesions that exclude any hereditary cause.

Transplant recipients and patients with childhood and adolescent cancers have also an increased risk of developing multiple NMSCs $[5,8,9]$. This is mainly due to the stringent chemotherapeutic regimen that diminishes the immuno-protective mechanisms and allows for neoplastic growth [6, 9]. Ionizing radiation therapy is also shown to cause a six-fold increase in developing NMSCs later in life [9]. None of these factors were identified when questioning the patient.

It is evident in our case that the patient had no known comorbidities or other predisposing factors other than chronic sun exposure that could influence his current clinical profile. Neglect could be a possible cause of these simultaneous SCC and BCC lesions, suggested by the patient identifying them a decade prior to his visit to the clinic. This is commonly reported in patients with poor medical knowledge and perhaps the slow growth with prolonged phenotypic changes may not have caused much concern for the patient to seek medical care [10].

There is also data to suggest that there is an increased risk in developing a subsequent cancer after the initial primary NMSC [11]. Combining all these possible factors, we propose the 

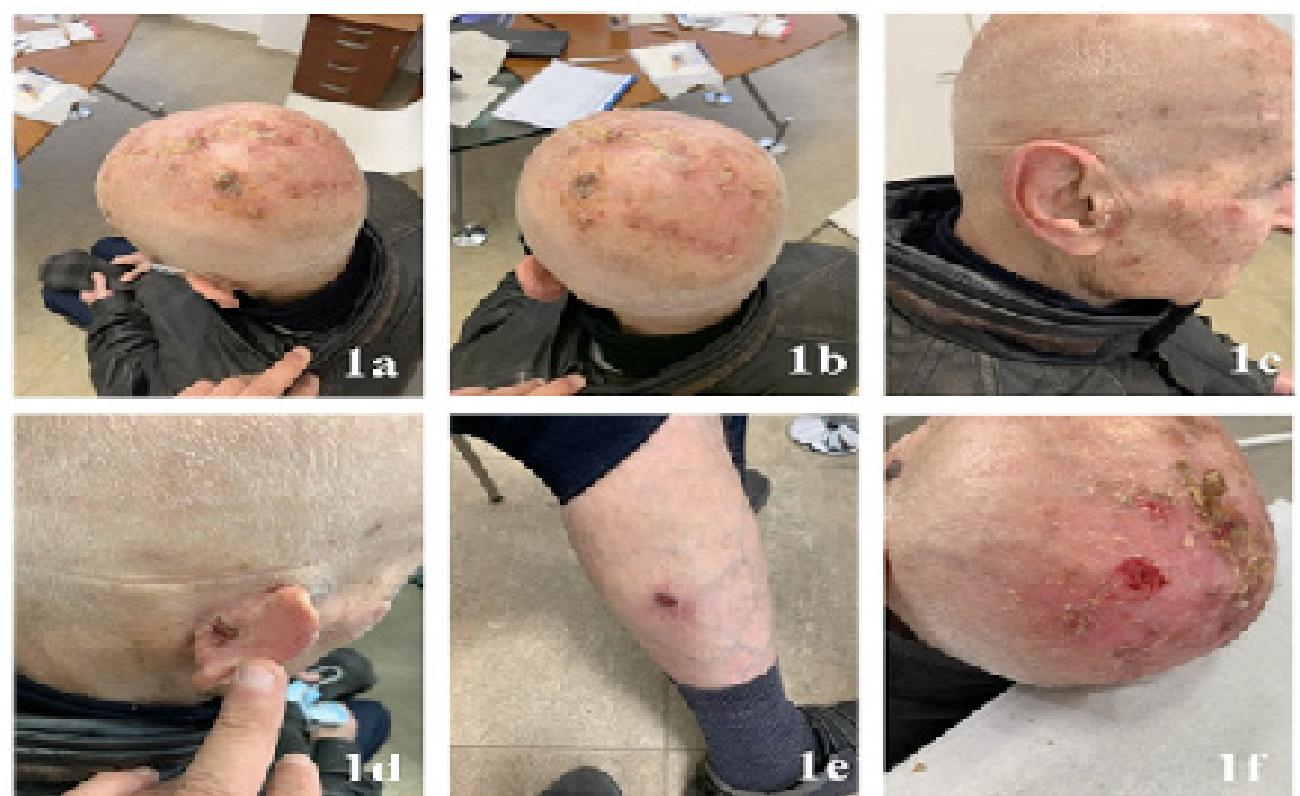

Fig. 1a, 1b, 1c: Patient with multiple actinic keratoses localised in areas of the mid-scalp, crown and preauricular regions.

Fig. 1d: Periauricular localised actinic keratosis.

Fig. 1e: Oval epithelial tumour in the area of the right lower leg subsequently identified as basal cell carcinoma, clear resection margins.

Fig. 1f: Epithelial tumour, with an erosive surface and relatively clear distinction from healthy tissue in the area of the scalp. The lesion was verified by histopathology as squamous cell carcinoma of the skin.
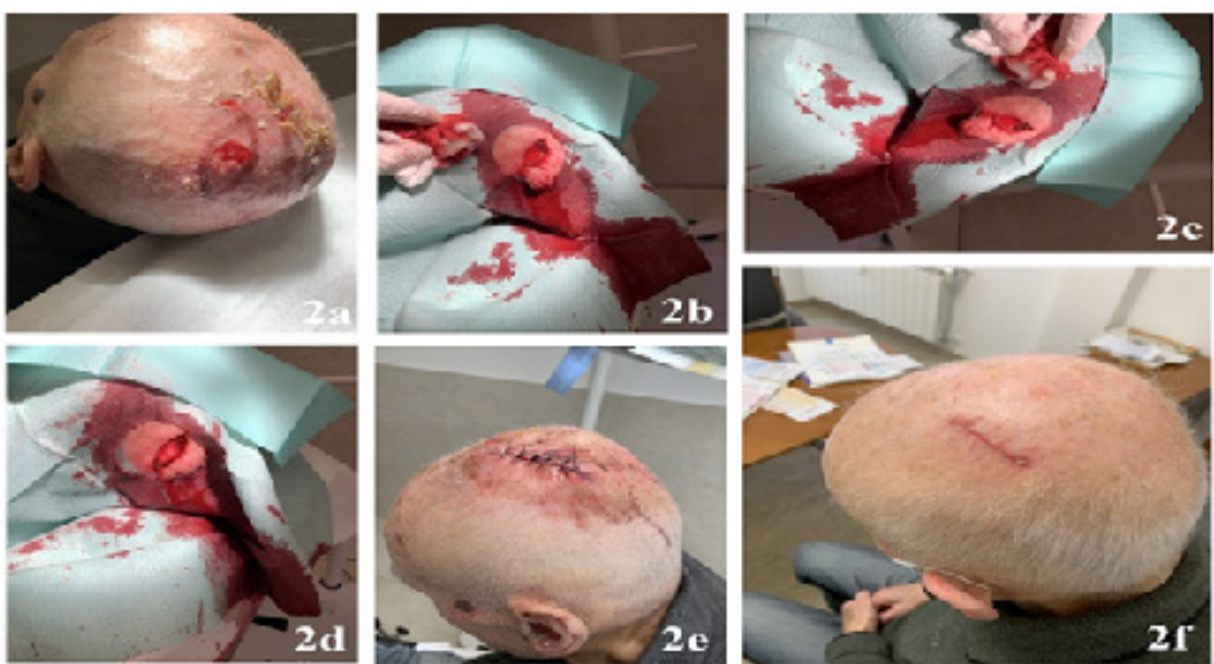

Fig. 2a: Resection marking around the epithelial tumour, localised in the area of the hairless part of the scalp.

Fig. 2b, c, d: Gradual excision of the tumour formation in depth in the form of a leaf-shaped surgical incision. Bleeding was controlled with an electrocautery knife.

Fig. 2e: Closing the defect by means of single skin sutures. Postoperative finding.

Fig. 2f: Day 21 postoperative clinical finding.

Citation: Oliveira N, Tchernev G (2021). Simultaneous Development of SCC of the head and BCC of the leg: Successful Surgical Approach in Bulgarian patient. Clin Res Dermatol Open Access 8(1): 1-3. DOI: 10.15226/2378-1726/8/1/001131 
possibility of a complex etiopathogenesis that includes chronic sunlight exposure, a longer period of time for multiple tumour growth together with neglect, and the increased risk factor of developing a second primary lesion distant to the former SCC lesion.
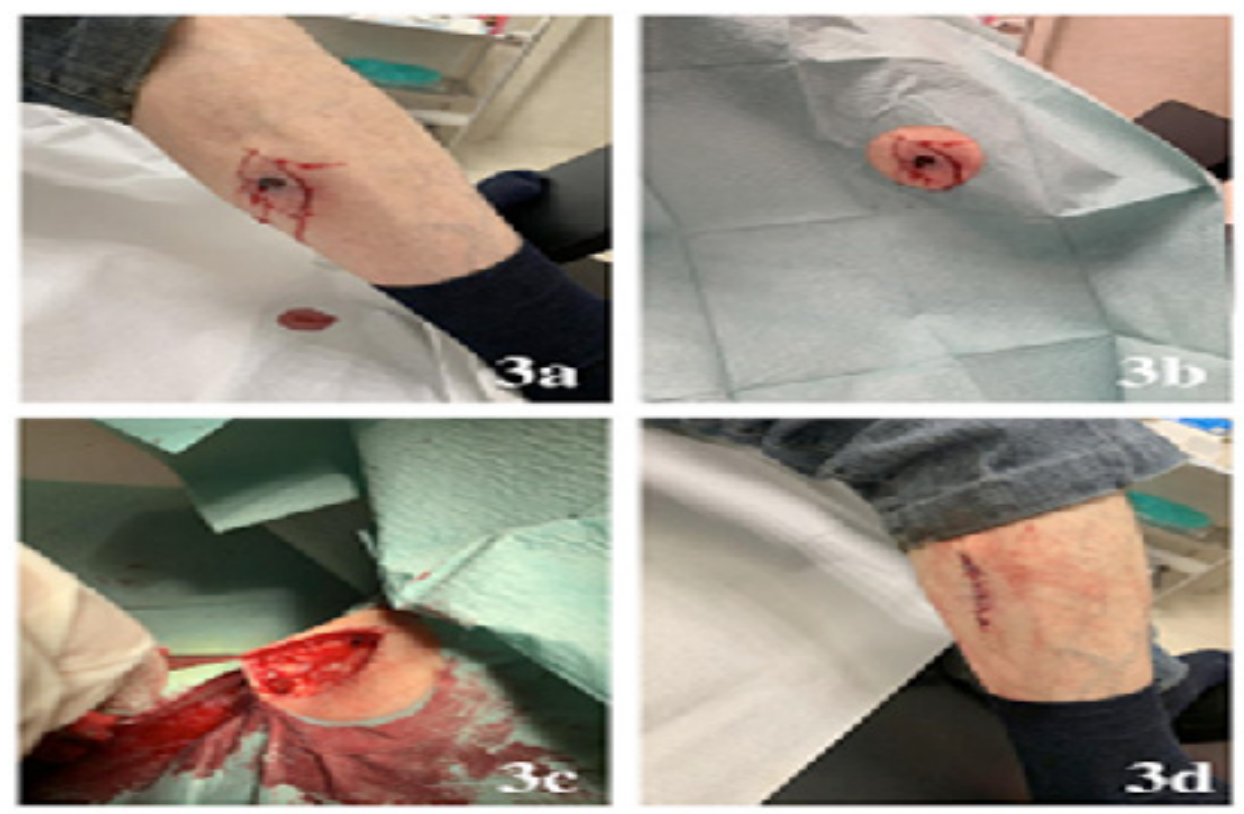

Fig. 3a, b: Marking of resection lines and placement of local anaesthesia with lidocaine.

Fig. 3c: Intraoperative finding after tumour removal and preparation of the peripheral skin in order to achieve optimal mobility so that the defect can be closed by means of extensible plastic.

Fig. 3d: Postoperative finding after adaptation of the wound edges and closure of the defect by means of single skin sutures.

\section{References}

1. Ferlay J, Colombet M, Soerjomataram I, Mathers C, Parkin D, Pineros M, et al. Estimating the global cancer incidence and mortality in 2018: GLOBOCAN sources and methods. Int J Cancer. 2019;144(8):19411953.doi:10.1002/ijc.31937.

2. Iannacone M, Wang W, Stockwell H, O'Rourke K, Giuliano A, Sondak $\mathrm{V}$, et al. Patterns and timing of sunlight exposure and risk of basal cell and squamous cell carcinomas of the skin-a case-control study. BMC Cancer. 2012; 12: 417.

3. Benjamin C and Ananthaswamy H. p53 and the pathogenesis of skin cancer. Toxicol Appl Pharmacol.2007;224(3):241-248.doi: 10.1016/j. taap.2006.12.006.

4. Benjamin C, Melnikova V, Ananthaswamy. P53 protein and pathogenesis of melanoma and nonmelanoma skin cancer. Adv Exp Med Biol. 2008;624:265-282.doi:10.1007/978-0-387-77574-6_21.

5. Pereira S, Curado M, Ribeiro A. Multiple skin neoplasms in subjects under 40 years of age in Goiania, Brazil.Rev Saude Publica. 2015;49:64. doi:10.1590/S0034-8910.2015049005777.

6. Awan B, Alzanbagi H, Samargandi O, Ammar H. Scalp squamous cell carcinoma in xeroderma pigmentosum. N Am J Med Sci.2014; 6(2):105-106.doi:10.4103/1947-2714.127754.

7. Emir S, Hacisalihoglu S, Ozyoruk D, Kacar D, Erdem A, Karakus E. Squamous cell carcinoma associated with Xeroderma pigmentosum: an unsual presentation with a tremendously huge mass over the face and paraneoplastic hypercalcemia - hyperleukocytosis. Turk J
Pediatr.2017;59(6):711-714.doi:10.24953/turkjped.2017.06.017.

8. Mittal A and Colegio O. Skin cancers in organ transplant recipients. Am J Trans. 2017; 17 (10): 2509-2530. doi: 10.1111/ajt.14382.

9. Perkins J, Liu Y, Mitby P, Neglia J, Hammond S, Stovall M, et al. Nonmelanoma skin cancer in survivors of childhood and adolescent cancer: A report from the childhood cancer survivor study. J Clin Oncol.2005;23(16):3733-3741.doi:10.1200/JC0.2005.06.237.

10. Varga E, Korom I, Rasko Z, Kis E, Varga J, Olah J, et al. Neglected basal cell carcinomas in the $21^{\text {st }}$ century. J Skin Cancer. 2011; 2011: 392151. doi:10.1155/2011/392151.

11. Rees J, Zens M, Gui J, Celaya M, Riddle B, Karagas M. Non melanoma skin cancer and subsequent cancer risk. PLoS ONE 2014;9(6): doi:10.1371/journal.pone.0099674. 